OPEN ACCESS

Edited by: Isadora Olivé, Université Vincennes Saint Denis Paris 8, France

Reviewed by: Philip R. Corlett, Yale University, USA Matthew W. Johnson, Johns Hopkins University, USA

${ }^{*}$ Correspondence: Matthew M. Nour matthew.nour@kcl.ac.uk

Received: 18 February 2016 Accepted: 20 May 2016 Published: 14 June 2016

Citation: Nour MM, Evans L, Nutt D and Carhart-Harris RL (2016) Ego-Dissolution and Psychedelics: Validation of the Ego-Dissolution Inventory (EDI).

Front. Hum. Neurosci. 10:269. doi: 10.3389/fnhum.2016.00269

\section{Ego-Dissolution and Psychedelics: Validation of the Ego-Dissolution Inventory (EDI)}

\author{
Matthew M. Nour ${ }^{1,2 *}$, Lisa Evans ${ }^{3}$, David Nutt ${ }^{3}$ and Robin L. Carhart-Harris ${ }^{3}$ \\ ${ }^{1}$ Psychiatric Imaging Group, MRC Clinical Sciences Centre, Imperial College London, London, UK, ${ }^{2}$ Institute of Psychiatry \\ Psychology and Neuroscience, King's College London, London, UK, ${ }^{3}$ Faculty of Medicine, Centre for \\ Neuropsychopharmacology, Division of Brain Sciences, Imperial College London, London, UK
}

Aims: The experience of a compromised sense of "self", termed ego-dissolution, is a key feature of the psychedelic experience. This study aimed to validate the Ego-Dissolution Inventory (EDI), a new 8-item self-report scale designed to measure ego-dissolution. Additionally, we aimed to investigate the specificity of the relationship between psychedelics and ego-dissolution.

Method: Sixteen items relating to altered ego-consciousness were included in an internet questionnaire; eight relating to the experience of ego-dissolution (comprising the EDI), and eight relating to the antithetical experience of increased self-assuredness, termed ego-inflation. Items were rated using a visual analog scale. Participants answered the questionnaire for experiences with classical psychedelic drugs, cocaine and/or alcohol. They also answered the seven questions from the Mystical Experiences Questionnaire (MEQ) relating to the experience of unity with one's surroundings.

Results: Six hundred and ninety-one participants completed the questionnaire, providing data for 1828 drug experiences (1043 psychedelics, 377 cocaine, 408 alcohol). Exploratory factor analysis demonstrated that the eight EDI items loaded exclusively onto a single common factor, which was orthogonal to a second factor comprised of the items relating to ego-inflation (rho $=-0.110$ ), demonstrating discriminant validity. The EDI correlated strongly with the MEQ-derived measure of unitive experience (rho $=0.735$ ), demonstrating convergent validity. EDI internal consistency was excellent (Cronbach's alpha 0.93). Three analyses confirmed the specificity of ego-dissolution for experiences occasioned by psychedelic drugs. Firstly, EDI score correlated with drug-dose for psychedelic drugs (rho $=0.371$ ), but not for cocaine (rho $=0.115$ ) or alcohol ( $r h o=-0.055)$. Secondly, the linear regression line relating the subjective intensity of the experience to ego-dissolution was significantly steeper for psychedelics (unstandardized regression coefficient $=0.701$ ) compared with cocaine $(0.135)$ or alcohol (0.144). Ego-inflation, by contrast, was specifically associated with cocaine experiences. Finally, a binary Support Vector Machine classifier identified experiences occasioned by psychedelic drugs vs. cocaine or alcohol with over $85 \%$ accuracy using ratings of ego-dissolution and ego-inflation alone.

Conclusion: Our results demonstrate the psychometric structure, internal consistency and construct validity of the EDI. Moreover, we demonstrate the 
close relationship between ego-dissolution and the psychedelic experience. The EDI will facilitate the study of the neuronal correlates of ego-dissolution, which is relevant for psychedelic-assisted psychotherapy and our understanding of psychosis.

\section{Keywords: ego dissolution, ego disintegration, ego death, psychedelic, self disturbance, ego boundaries, mystical} experience, hallucinogen

\section{INTRODUCTION}

Distortions in the subjective experience of one's "self", or "ego", are central to the psychedelic experience (James, 1882; Huxley, 1954; Savage, 1955; Klee, 1963; Leary et al., 1964; Grof, 1976, 1980; Harrison, 2010; Carhart-Harris et al., 2014; Lebedev et al., 2015). Specifically, a reduction in the self-referential awareness that defines normal waking consciousness has been reported with all classical psychedelic drugs (5- $\mathrm{HT}_{2 \mathrm{~A}}$ receptor agonists), including psilocybin (Griffiths et al., 2008, 2011), lysergic acid diethylamide (LSD; Goodman, 2002; Lyvers and Meester, 2012), and dimethyltryptamine (DMT; Trichter et al., 2009), as well as with other psychoactive substances such as nitrous oxide (James, 1882) and ketamine (Vollenweider and Kometer, 2010).

The experience of a compromised sense of self occasioned by psychedelic drugs has been variously called ego-death (Grof, 1980; Harrison, 2010), ego-loss (Leary et al., 1964), egodisintegration (Muthukumaraswamy et al., 2013; Lebedev et al., 2015) and ego-dissolution (Klee, 1963; Studerus et al., 2010; Carhart-Harris et al., 2014; Lebedev et al., 2015; Tagliazucchi et al., 2016). This experience has been interpreted from a psychoanalytic perspective as a disruption of ego-boundaries, which results in a blurring of the distinction between selfrepresentation and object-representation, and precludes the synthesis of self-representations into a coherent whole (Federn, 1952; Savage, 1955; Fischman, 1983).

It is likely that the prior "psychology" of the subject and the environmental setting in which they take a psychedelic influences whether an ego-dissolution experience is welcomed and felt as something positive, or feared and fought against (Eveloff, 1968; Fischman, 1983; Griffiths et al., 2008; Studerus et al., $2010,2012)$. At one extreme, ego-dissolution is closely related to blissful mystical experiences such as can be occasioned by certain spiritual or religious practices (Stace, 1960; Hood, 1975; MacLean et al., 2012); indeed, loss-of-self was identified by William James as being a cardinal feature of the mystical experience (James, 1985). These experiences are characterized by a feeling of unity with one's surroundings, which is explicitly related to disturbed ego-boundaries and thus ego-dissolution. Moreover, the mystical experience is likely to be of therapeutic benefit in psychedelicassisted psychotherapy (Leary et al., 1964; Grof, 1980; Griffiths et al., 2008, 2011; Johnson et al., 2008, 2014). At the other extreme, it has been argued that self-disturbances and disturbed ego-boundaries are a core phenomenological feature of psychosis and schizophrenia (Bleuler, 1950; Laing, 1959; Scharfetter, 1981; Fischman, 1983; Parnas, 2011; Sass et al., 2011; Northoff, 2014; Nour and Barrera, 2015), although it remains unclear precisely how the self-disturbances specific to schizophrenia relate to the experience of ego-dissolution under psychedelics.
Discussions of altered self-experience have been traditionally confined to philosophy or descriptive psychopathology (Stace, 1960; Jaspers, 1997). In recent years, however, there has been increased interest in the neurobiological correlates of the experience of self (Carhart-Harris and Friston, 2010; Qin and Northoff, 2011; Carhart-Harris et al., 2014). Psychedelic drugs may provide a fruitful avenue of research into the neuronal correlates of normal and abnormal self-awareness or ego-consciousness (Carhart-Harris et al., 2013, 2014; Muthukumaraswamy et al., 2013; Roseman et al., 2014; Lebedev et al., 2015; Tagliazucchi et al., 2016). This research programme, however, is predicated on the existence of a validated self-report measure of the ego-dissolution experience.

There currently exist several measures that capture feelings related to alterations in self-experience (Strassman et al., 1994; Parnas et al., 2005; Studerus et al., 2010; MacLean et al., 2012). Dittrich's APZ (Abnormal Mental States) questionnaire, and its revised versions, OAV and 5D-ASC, have been used extensively to characterize altered states of consciousness occasioned by psychedelic drugs (Dittrich, 1998; Studerus et al., 2010). These questionnaires purport to capture both positive and negative experiences of depersonalization and derealization ("oceanic boundlessness" and "dread of ego dissolution", respectively), as well as additional dimensions of "visionary restructuralization" (in APZ, OAV and 5D-ASC), "auditory alterations" and "vigilance reductions" (both in 5D-ASC only; Dittrich, 1998; Hasler et al., 2004; Wittmann et al., 2007; Studerus et al., 2010, 2011; Schmid et al., 2015). Recent psychometric evaluation of the OAV questionnaire, however, reveals a more complex 11 -factor structure, including factors relating to changes in cognition, perception and mood, as well as to feelings of unity and disembodiment. At present, however, there are no validated scales that allow an easy, reliable and direct uni-dimensional measurement of egodissolution. This presents a barrier to this promising line of research.

The primary aim of the present study was to develop and validate the "Ego-Dissolution Inventory" (EDI), a new succinct 8 -item self-completed questionnaire designed to operationalize the experience of ego-dissolution so that its construct validity can be tested and developed. In order to do this in an efficient way we chose to utilize online data collection via a large anonymous internet survey. A secondary aim was to investigate the specificity of the relationship between the experience of ego-dissolution and psychedelic drugs, compared with cocaine and alcohol. These two comparator drugs were chosen because of their widespread availability and use in Western societies. Finally, we aimed to test the hypothesis that experiences occasioned by classical stimulant drugs, like cocaine, are in some respects antithetical to the 
psychedelic experience, promoting ego-inflation rather than egodissolution.

\section{MATERIALS AND METHODS}

\section{Survey Construction}

\section{Ego-Dissolution Inventory Item Selection}

Sixteen novel statements relating to the experience of ego-consciousness were included in this study. Eight of these were designed to capture the central phenomenon of egodissolution (and the associated feeling of increased union with one's surroundings, known as dissolved ego-boundaries), and particularly how it has been characterized in the context of the psychedelic experience (Leary et al., 1964; Grof, 1980; Harrison, 2010). As well as referring to the existing literature on the psychedelic experience, we also sought the opinion of six scientists working within the field of psychedelic neuroscience when choosing the final eight ego-dissolution items, and sought consensus about the chosen items. The other eight items were designed to reflect the distinct and largely antithetical experience of unusually elevated self-assuredness and confidence (which we refer to as "ego-inflation"). The items from these two subscales were included in the final survey in an interleaved manner with the intention of minimizing question "order" effects, and the potential tendency for subjects to indiscriminately endorse any statement about altered consciousness when reflecting on an experience with a psychoactive substance (particularly if that substance can have profound and variegated psychological effects, as is the case with psychedelics). Items were rated using a visual analog scale format (0-100, with incremental units of one) with zero defined as "No, not more than usually", and 100 defined as "Yes, entirely or completely", taking inspiration from a previous questionnaire on altered states of consciousness developed by Dittrich (1998) as well as other self-constructed scales used internally by our team (Carhart-Harris et al., 2012, 2015; Muthukumaraswamy et al., 2013). For the exact wording of the 16 ego-consciousness items included in the survey see Table 1.

\section{Survey Structure}

Each subject was asked to provide information on their age, sex and educational background. Subjects also provided information on their lifetime use of psychedelic drugs and cocaine, as well as their weekly alcohol consumption (all possible answer options for educational attainment and drug and alcohol use are presented in Table 2).

After providing these demographic data, subjects were given the opportunity to answer questions on up to four drug experiences: (1) their "most intense" psychedelic experience; (2) a "typical" psychedelic experience; (3) a "typical" cocaine experience; and (4) a "typical" alcohol experience. For psychedelic experiences subjects could further specify the drug taken (options were: LSD, psilocybin, mescaline, DMT and ayahuasca). For each experience subjects were asked to provide information on how long ago the experience was (options were: “Today", "Last week", "1-4 weeks" ago, "1-6
TABLE 1 | Factor loadings from exploratory factor analysis of item scores from 1828 drug experiences (extraction method: principal axis factor; rotation method: promax with Kaiser normalization; loadings taken from pattern matrix)

\begin{tabular}{|c|c|c|}
\hline Item: & $\begin{array}{c}\text { Factor } 1 \\
\text { Ego-dissolution }\end{array}$ & $\begin{array}{c}\text { Factor } 2 \\
\text { Ego-inflation }\end{array}$ \\
\hline I felt especially assertive & 0.014 & 0.691 \\
\hline $\begin{array}{l}\text { I experienced a dissolution } \\
\text { of my "self" or ego }\end{array}$ & 0.883 & -0.040 \\
\hline $\begin{array}{l}\text { I felt more important or special } \\
\text { than others }\end{array}$ & -0.034 & 0.715 \\
\hline $\begin{array}{l}\text { I felt at one with } \\
\text { the universe }\end{array}$ & 0.830 & 0.010 \\
\hline My ego felt inflated & -0.154 & 0.756 \\
\hline $\begin{array}{l}\text { I felt a sense of union } \\
\text { with others }\end{array}$ & 0.700 & 0.167 \\
\hline I felt especially sure-of-myself & 0.151 & 0.824 \\
\hline $\begin{array}{l}\text { I experienced a decrease } \\
\text { in my sense of self-importance }\end{array}$ & 0.663 & -0.066 \\
\hline $\begin{array}{l}\text { I felt especially keen and } \\
\text { competitive }\end{array}$ & -0.134 & 0.725 \\
\hline $\begin{array}{l}\text { I experienced a disintegration } \\
\text { of my "self" or ego }\end{array}$ & 0.897 & -0.070 \\
\hline $\begin{array}{l}\text { I felt like my viewpoint was worth more } \\
\text { than other peoples' }\end{array}$ & -0.075 & 0.667 \\
\hline $\begin{array}{l}\text { I felt far less absorbed by my } \\
\text { own issues and concerns }\end{array}$ & 0.624 & 0.112 \\
\hline I felt especially self-confident & 0.073 & 0.847 \\
\hline I lost all sense of ego & 0.885 & -0.043 \\
\hline I felt especially self-assured & 0.156 & 0.822 \\
\hline $\begin{array}{l}\text { All notion of self and identity } \\
\text { dissolved away }\end{array}$ & 0.845 & -0.042 \\
\hline
\end{tabular}

Items in bold comprise the Ego-Dissolution Inventory (EDI), whilst the remaining items relate to ego-inflation. All factor loadings $>0.2$ are in bold.

months" ago, "6-12 months" ago, " $1-5$ years" ago, "6-10 years" ago, and "Over 10 years" ago). They were also asked how "intense" the experience was (for psychedelic experiences), or how "energized/wired" or "inebriated/drunk" they felt (for cocaine and alcohol experiences, respectively) on a visual analog scale from 0 to 100 , with $0=$ "Not at all" and $100=$ "The most intense/energized/inebriated imaginable". The rationale for enquiring about a typical and most intense experience with psychedelics was to collect a greater range of possible responses with regards to psychedelics, which was our primary drug class of interest.

For each experience subjects were also asked to state the dose of the drug taken. For psychedelic drugs, subjects were asked to provide a "rough/ballpark" estimate using an LSDequivalent dose; available options ranged from "No more than half a tab/50 micrograms of LSD" to "More than 3 tabs/300 micrograms of LSD", split into 5 non-overlapping groups. This was done with the aim of providing a standard reference against which any non-LSD classical psychedelic could be compared. For cocaine, the dose options available ranged from: "less than $1 / 8$ gram" to "More than 2 grams", split into six 
TABLE 2 | Demographic data for subjects who provided information for at least one drug experience.

\begin{tabular}{|c|c|c|}
\hline Total & 691 & \\
\hline Female & 238 (34.44\%) & \\
\hline \multicolumn{3}{|l|}{ Age at time of survey } \\
\hline Median & 28 & \\
\hline Inter-quartile range & 13 & \\
\hline Skewness & 1.43 & \\
\hline \multicolumn{3}{|l|}{ Education } \\
\hline Left school before age 16 (no qualifications) & $2(0.29 \%)$ & \\
\hline Left school at 16/GCSE (UK) & 29 (4.20\%) & \\
\hline High school diploma/A-Level (UK) & $68(9.84 \%)$ & \\
\hline Some university (or equivalent) & $168(24.31 \%)$ & \\
\hline Bachelor's degree (or equivalent) & 229 (33.14\%) & \\
\hline Post-graduate degree (or equivalent) & $195(28.22 \%)$ & \\
\hline Lifetime illicit drug use & Psychedelic & Cocaine \\
\hline Never & $107(15.48 \%)$ & $236(34.15 \%)$ \\
\hline Once only & $26(3.76 \%)$ & $70(10.13 \%)$ \\
\hline $2-5$ times & $106(15.34 \%)$ & $106(15.34 \%)$ \\
\hline $6-10$ times & $92(13.31 \%)$ & 59 (8.54\%) \\
\hline $11-15$ times & 69 (9.99\%) & 35 (5.07\%) \\
\hline 16-25 times & 69 (9.99\%) & $45(6.51 \%)$ \\
\hline 26-50 times & 87 (12.59\%) & $42(6.08 \%)$ \\
\hline $51-100$ times & 68 (9.84\%) & 47 (6.80\%) \\
\hline$>100$ times & $67(9.70 \%)$ & $51(7.38 \%)$ \\
\hline
\end{tabular}

Weekly alcohol consumption

No alcohol

$1-6$ units

7-12 units

13-18 units

19-24 units

25-30 units

31-36 units

37-42 units

43-48 units

49-54 units

55-60 units

$>60$ units to load onto a single common factor denoting "Mystical" experiences (Barrett et al., 2015), and all relate to the so-called "unitive" experience, which is considered to be a fundamental feature of the mystical experiences (Stace, 1960; Hood, 1975; James, 1985). The unitive experience is related to the notion of dissolved ego-boundaries, and it has been hypothesized (although never formally investigated) that the phenomenology of the unitive experience overlaps with that of ego-dissolution (James, 1882; Leary et al., 1964; Grof, 1980; Harrison, 2010). The inclusion of the relevant MEQ questions in this survey allowed us to explicitly test this hypothesis, and provided a means of measuring the convergent validity of the ego-dissolution construct.

The specific MEQ questions included, and their relevant MEQ30 identifiers (Barrett et al., 2015), were as follows: "Freedom from the limitations of your personal self and feeling a unity or bond with what was felt to be greater than your personal self" [MEQ30 Q14], "Experience of oneness in relation to an 'inner world' within" [MEQ30 Q20], "Experience of the fusion of your personal self into a larger whole" [MEQ30 Q26], "Experience of unity with ultimate reality” [MEQ30 Q28], "Feeling that you experienced eternity or infinity" [MEQ30 Q05], "Experience of oneness or unity with objects and/or persons perceived in your surroundings" [MEQ30 Q06] and "Experience of the insight that 'all is One"" [MEQ30 Q18]. Each item was rated on a 6-point scale, where $0=$ "none, not at all"; $1=$ "so slight cannot decide"; 2 = "slight"; 3 = "moderate"; 4 = "strong (equivalent in degree to any previous strong experience or expectation of this description)"; and $5=$ "extreme (more than ever before in my life and stronger than four)". This is consistent with the standard procedure for completion of the MEQ.

\section{Dissemination of the Survey}

This study was approved by the local ethics committee. The survey was implemented and hosted by the online service Survey Gizmo $^{1}$, and was estimated to take 38 min to complete. Survey Gizmo has comprehensive privacy policies and security features that maintain the anonymity of responses in line with ethics requirements.

Participants were recruited to take the online survey via weblink advertisements posted on Facebook groups, Twitter pages, email newsletters, and online drug forums with a short request ("please participate in our anonymous online questionnaire designed to learn more about experiences with classical psychedelics, cocaine, and alcohol"). Recruitment targeted online communities interested in psychoactive substances and altered states of consciousness (e.g., Psychedelic Society ${ }^{2}$, and Multidisciplinary Association for Psychedelic Studies ${ }^{3}$ ), as well as websites visited by more diverse populations, (e.g., Reddit ${ }^{4}$, and Mumsnet ${ }^{5}$ ). The collection of IP addresses and geographical locations of participants was disabled and participants were informed of the anonymity of their responses.

\footnotetext{
${ }^{1}$ http://www.surveygizmo.eu/

${ }^{2}$ http://www.psychedelicsociety.org.uk

${ }^{3}$ http://www.maps.org

${ }^{4}$ https://www.reddit.com/

${ }^{5}$ http://www.mumsnet.com/
} 
After reading a summary of the inclusion criteria and instructions, participants provided informed consent by clicking "next" on the first page of the questionnaire.

Inclusion criteria for participants were: (1) at least 18 years of age; and (2) had had at least one experience with a classical psychedelic (LSD, psilocybin, DMT, ayahuasca or mescaline), cocaine, and/or alcohol. Data collection occurred over a 4-week period.

\section{Statistical Analysis}

\section{Factor Analysis and Definition of Ego-Dissolution Inventory}

We defined a completed form as one in which the subject had answered all 16 ego-consciousness items relating to at least one drug experience, and also provided information about the dose of drug taken, the subjective intensity of the experience, and the effect on well-being.

Scores for the 16 ego-consciousness items for each complete form were subjected to an exploratory factor analysis using the iterated principle axis factor method and an oblique (promax) rotation, which allowed common factors to be correlated (Budaev, 2010). The appropriate number of factors to be extracted was determined by parallel analysis of principal components using 1000 random draws (Horn, 1965; O'Connor, 2000) and Cattell's scree plot criterion (Cattell, 1966). Based on the factor loadings (from the pattern matrix), the 16 ego-consciousness items could be easily separated into two 8 -item scales reflecting "ego-dissolution" and "ego-inflation" experiences (full details in "Results" Section). The mean item scores for both the ego-dissolution and ego-inflation scales were used as a measure of ego-dissolution and ego-inflation for all subsequent analysis.

\section{Reliability and Construct Validity}

Internal consistency of the scales was assessed with Cronbach's alpha (Cronbach, 1951). Convergent validity of the egodissolution scale was assessed using the correlation with our MEQ-derived measure of unitive experience for psychedelic experiences. Discriminant validity was demonstrated firstly by the ability of an exploratory factor analysis to separate the eight ego-dissolution items from the eight ego-inflation items, and secondly by demonstrating the specificity of ego-dissolution for the psychedelic experience over alcohol or cocaine experiences (see "Relationship of Ego-Dissolution to Psychedelic Drugs and Persisting Effects" Section).

\section{Relationship of Ego-Dissolution to Psychedelic Drugs and Persisting Effects}

The specificity of the relationship between ego-dissolution and experiences occasioned by classical psychedelics was tested in three ways. Firstly we investigated the correlation between reported drug dose and both ego-dissolution and ego-inflation for each drug class separately (where drug dose was defined as the central value for the selected dose range for each experience). For each drug class the null hypothesis that the dose-ego-dissolution and dose-ego-inflation correlations were equal was tested using a 2-tailed $t$-test of the differences between dependent correlation coefficients (Field, 2013).

Secondly, we investigated the relationship between the reported subjective intensity of the experience and egodissolution. Correlations between experience intensity and ego-experiences within a drug class were investigated in an identical manner to the dose-response relationships. As ratings of subjective experience intensity are theoretically comparable across drug classes (unlike drug doses) we were able to test the hypothesis that the linear relationship between subjective intensity and ego-dissolution (or ego-inflation) was different between psychedelic, cocaine and alcohol experiences by testing whether the slope of the linear regression line relating subjective intensity (independent variable) to ego-dissolution or egoinflation (dependent variables) is significantly different between drug classes (using MATLAB's aoctool [Analysis of covariance (ANCOVA)] function [MATLAB 2015b, Mathworks]).

Finally, a Support Vector Machine (SVM) classifier (a common supervised machine learning algorithm) was trained to distinguish between typical experiences with psychedelics, cocaine and alcohol using only the ego-dissolution and egoinflation score for each experience, in three binary one-v-one classification tasks (psychedelic vs. cocaine, psychedelics vs. alcohol, cocaine vs. alcohol). Only "typical" psychedelic experiences were included in this analysis to avoid classification problems associated with numerically imbalanced classes $(\mathrm{He}$ and Garcia, 2009). The SVM classifier was implemented in MATLAB 2015b (Mathworks), as part of the Classification Learner application, with the following settings: 5-fold crossvalidation, linear kernel, standard normalization transformation applied to data before entering into SVM classifier.

The correlation between ego-dissolution or ego-inflation and reported changes in personal well-being was compared both between and within drug classes using Fisher's $r$-to- $z$ transform and 2-tailed $t$-test, respectively (Field, 2013).

Spearman's rho was used to quantify all bivariate correlations. Statistical significance is defined as $p<0.05$ (two-tailed). Multiple statistical comparisons were performed when analyzing the correlation between ego-dissolution or ego-inflation and variables of interest (e.g., drug dose, experience intensity, or change in well-being) separately for the three drug classes. In these instances, we applied Bonferroni correction for multiple comparisons (specifically, six simultaneous comparisons) such that differences are deemed statistically significant for $p<0.008$. $95 \%$ confidence intervals were calculated using the bias corrected and accelerated bootstrap method (1000 samples). All statistical analysis was performed using SPSS Statistics (IBM, Version 22), and MATLAB (MathWorks, Version 2015b including Statistics and Machine Learning Toolbox).

\section{RESULTS}

\section{Baseline Demographics of Survey Responders}

Six-hundred and ninety-one subjects completed the online survey. Table 2 summarizes the demographic information 
for these subjects. Each subject answered questions for a mean of 2.65 drug experiences (SD 1.18), providing data for 1828 complete drug experiences for analysis (1043 were with psychedelic drugs: 584 relating to the most intense psychedelic experience and 459 relating to a typical psychedelic experience, occurring a median of 1-5 years and 6-12 months prior to survey completion, respectively. 377 were with cocaine, occurring a median of $1-5$ years prior to survey completion. 408 were with alcohol, occurring a median of 1-4 weeks prior to survey completion).

\section{Ego-Dissolution Inventory: Factor Structure and Internal Consistency}

To investigate the factor structure of the 16 ego-consciousness items in a hypothesis-free manner, all questions were submitted to an exploratory factor analysis. The Kaiser-Meyer-Olkin measure of sampling adequacy was 0.918 and Bartlett's test of sphericity was highly significant $\left(\chi_{(120)}^{2}=22441.7, p<0.001\right)$ confirming that the data were indeed suitable for factor analysis (Budaev, 2010).

Both parallel analysis for principle components (Horn, 1965; O'Connor, 2000) and inspection of the scree plot using Cattell's criterion (Cattell, 1966) supported a model with two factors or components (parallel analysis observed and 95\% confidence interval simulated eigenvalues for the 3 rd component were 1.04 and 1.12 , respectively). The first component explained $36.6 \%$ of the variance in the sample, and the second component explained $29.5 \%$ of the variance in the sample. All other components explained $<7 \%$ of the variance in the sample.

The data were therefore subjected to an exploratory factor analysis to extract two common factors. Factor 1 comprised of the eight items relating to the experience of "ego-dissolution" whilst Factor 2 comprised of the eight items relating to the experience of "ego-inflation". Communality values (the proportion of an item's variance that can be explained by the extracted common factors) ranged from $39 \%$ to $82 \%$. Every item loaded strongly and exclusively onto either Factor 1 or Factor 2, demonstrating a simple and easily interpretable factor structure (Table 1).

Guided by the results of the exploratory factor analysis, two 8 -item scales were derived, one reflecting the experience of "egodissolution", and one reflecting the experience of "ego-inflation" (Table 1). Both scales had excellent internal consistency (Cronbach's alpha $=0.93$ and 0.91 , respectively; Cronbach, 1951). The mean item scores for both the ego-dissolution and ego-inflation scale were almost perfectly correlated with the factor scores calculated using the regression method from the exploratory factor analysis output (both rho $>0.99, p<0.001$ ). For ease of interpretability and replication, the mean item scores from the two scales were used as a measure of ego-dissolution and ego-inflation for all subsequent analysis.

\section{Construct Validity}

For experiences with psychedelic drugs, the score for the MEQ-derived measure of unitive experience correlated strongly with ego-dissolution (rho $=0.735$ [95\% CI 0.704, 0.763], $p<0.001)$ providing a clear demonstration of convergent validity. This measure of unitive experience also correlated positively with ego-inflation (rho $=0.274$ [0.219, 0.332], $p<0.001$ ) but the strength of this correlation was significantly weaker than that between unitive experience and ego-dissolution $\left(t_{(1040)}=17.8, p<0.001\right)$. In the exploratory factor analysis, Factor 1 ("ego-dissolution") and Factor 2 ("ego-inflation") were essentially orthogonal (rho $=-0.11$ ), demonstrating the discriminant validity of ego-dissolution and ego-inflation subscales.

\section{Specificity of Ego-Dissolution for Psychedelic Drugs}

\section{Dose-Response Relationship}

For experiences with psychedelic drugs, there was a significant positive correlation between reported drug dose and egodissolution (rho $=0.371[0.317,0.427], p<0.001$ ), and only a weak correlation between reported drug dose and ego-inflation (rho $=0.063[0.003,0.127], p=0.043$ ), which did not survive correction for multiple comparisons (Figure 1A). The difference between these two correlations was significant $\left(t_{(1040)}=8.55\right.$, $p<0.001)$. Conversely, for cocaine, there was a significant and strong positive correlation between reported drug dose and ego-inflation (rho $=0.385[0.390,0.477], p<0.001$ ) but only a weak correlation between drug dose and ego-dissolution (rho $=0.115[0.012,0.211], p=0.026)$, which also did not survive correction for multiple comparisons (Figure 1B). Again, the difference between these two correlations was significant $\left(t_{(374)}=4.88, p<0.001\right)$. For alcohol there was no dose-response relationship with either ego-dissolution ( $r h o=-0.055[-0.150$, $0.048], p=0.266$ ) or ego-inflation (rho $=-0.054[-0.148$, 0.037 ], $p=0.328$ ), and no difference between these correlations $\left(t_{(405)}=0.031, p=0.488\right.$; Figure 1C).

\section{Intensity-Response Relationship}

For experiences with psychedelic drugs, the subjective intensity of the experience was positively correlated with both egodissolution (rho $=0.577[0.529,0.621], p<0.001$ ) and egoinflation (rho $=0.099[0.040,0.159], p=0.001$ ), although the correlation with ego-dissolution was significantly stronger than that with ego-inflation $\left(t_{(1040)}=15.1, p<0.001\right)$. The opposite pattern was observed for cocaine and alcohol experiences however, where there was a significantly stronger correlation between the subjective intensity of these drug experiences and ego-inflation (Cocaine: rho $=0.545$ [0.464, 0.625], $p<0.001$. Alcohol: $r h o=0.502[0.410,0.582], p<0.001)$ than egodissolution (Cocaine: rho $=0.279$ [0.180, 0.378], $p<0.001$. Alcohol: rho $=0.334[0.237,0.421], p<0.001) ;\left(t_{(374)}=5.33\right.$, $p<0.001$, and $t_{(405)}=4.03, p<0.001$, for cocaine and alcohol respectively).

ANCOVA analysis was used to fit separate regression lines relating subjective intensity (predictor variable) to egodissolution or ego-inflation (dependent variables), for each drug class separately. This analysis confirmed that ego-dissolution experiences were significantly predicted by experience intensity $\left(F_{(1,1822)}=528.4, \mathrm{MSE}=165132.7, p<0.001\right)$, drug class $\left(F_{(2,1822)}=636.39\right.$, MSE $\left.=198863.7, p<0.001\right)$, and 

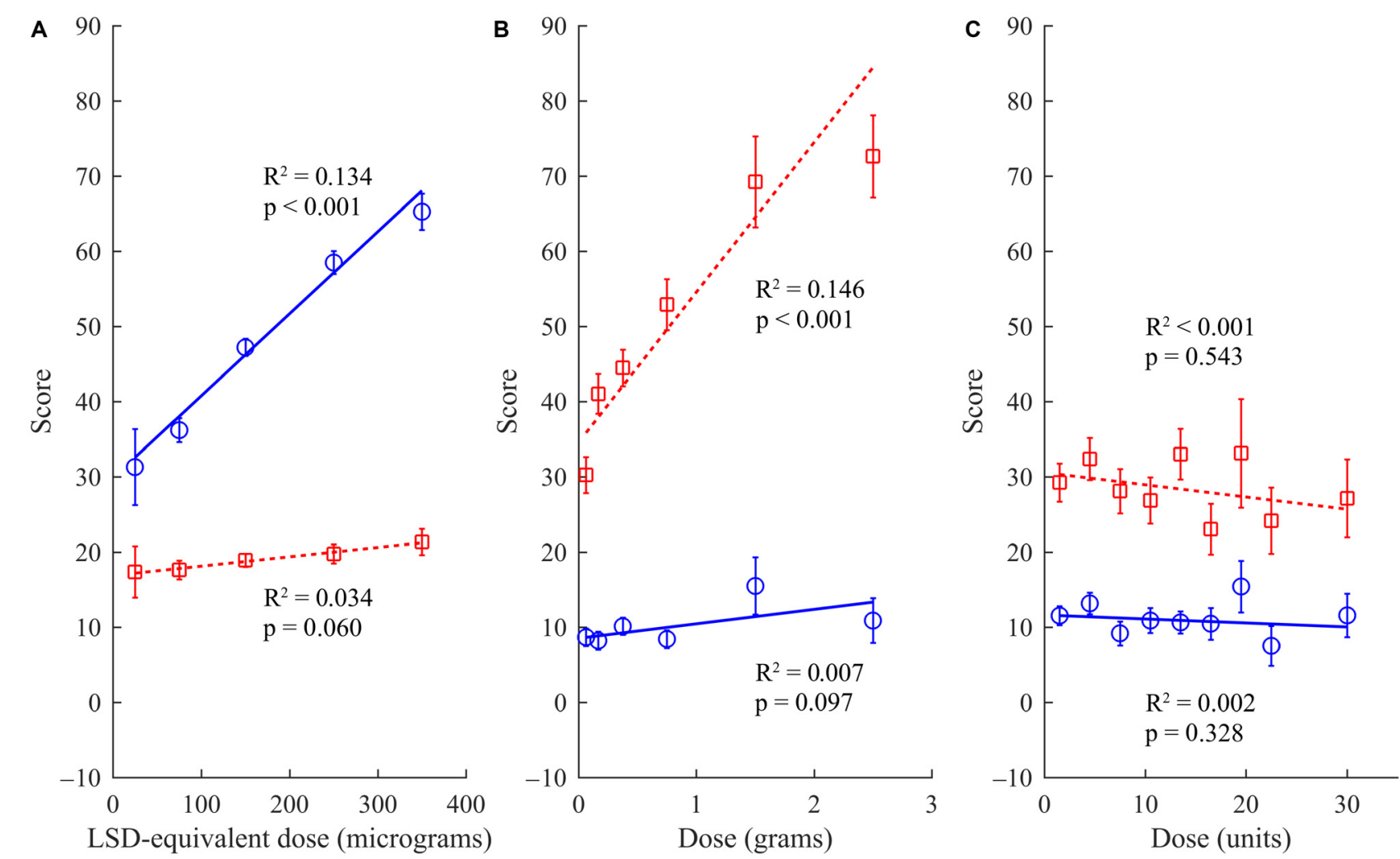

FIGURE 1 | Relationship between drug dose and scores for ego-dissolution (blue circles, solid line) and ego-inflation (red squares, dashed line) for experiences occasioned by different drug classes. (A) Psychedelic experiences $(n=1043)$. (B) Cocaine experiences $(n=377)$. (C) Alcohol experiences $(n=408)$. Lines represent linear regression lines of best fit, with corresponding $R^{2}$ and $p$-value. Error bars represent \pm 1 SEM.

the interaction between subjective intensity and drug class $\left(F_{(2,1822)}=116.29, \mathrm{MSE}=36338, p<0.001\right)$. Follow-up multiple comparison tests (Tukey's HSD criterion) demonstrated that the slope of the regression line relating experience intensity to ego-dissolution was significantly steeper for psychedelics (unstandardized regression coefficient $=0.701[0.640,0.762]$ ), than for cocaine $(0.135[0.088,0.182])$ or alcohol $(0.144$ [0.098, 0.191], both $p<0.001)$. There was no difference between the slopes of the regression lines relating experience intensity to ego-dissolution for alcohol vs. cocaine ( $p=0.984$; Figure 2A).

Ego-inflation experiences were also significantly predicted by subjective intensity $\left(F_{(1,1822)}=229.16\right.$, MSE $=89120.9$, $p<0.001)$, drug class $\left(F_{(2,1822)}=278.5, \mathrm{MSE}=108309.5\right.$, $p<0.001)$ and the interaction between drug class and intensity $\left(F_{(2,1822)}=61.16, \mathrm{MSE}=23785.8, p<0.001\right)$. The slope of the regression line relating experience intensity to ego-inflation, was, however, significantly shallower for psychedelics $(0.096$ [0.043, $0.149])$ compared with cocaine $(0.632[0.538,0.726], p<0.001)$ and alcohol $(0.439[0.357,0.521], p<0.001)$, and was steeper for cocaine than for alcohol experiences ( $p=0.003$; Figure $2 \mathbf{B}$ ).

\section{Support Vector Machine Classifier}

As a final demonstration of the specificity of the egodissolution experience for psychedelic drugs, we trained a
SVM binary classifier to distinguish between typical psychedelic $(n=459)$, cocaine $(n=377)$ and alcohol $(n=408)$ drug experiences using only the ego-dissolution and egoinflation scores. This classifier achieved an accuracy of 90.1\% when distinguishing between psychedelic vs. cocaine experiences (receiver-operator characteristic (ROC) area under the curve (AUC) = 0.958), and 85.2\% accuracy when distinguishing between psychedelic and alcohol experiences (ROC AUC $=0.927$ ). By contrast, it performed poorly when distinguishing cocaine from alcohol experiences $(63.4 \%$ accuracy, ROC AUC $=0.685)$. Classification at chance level is equivalent to $50 \%$ accuracy and ROC AUC $=0.5$. Figure 3 illustrates the relationship between ego-dissolution and ego-inflation for experiences occasioned by different drugs.

\section{Ego-Dissolution and Well-Being}

As a final exploratory analysis, we sought to investigate the relationship between ego-experiences and the extent to which the experience in question changed subjects' current sense of personal well-being or life satisfaction. For psychedelic drugs, the median response on the 7 -point rating scale (with possible answers from -3 to +3$)$ was $+2(+2=$ "Increased moderately", interquartile range $=2$, skewness $=-0.769$, median time elapsed since experience $=1-5$ years), which was significantly 


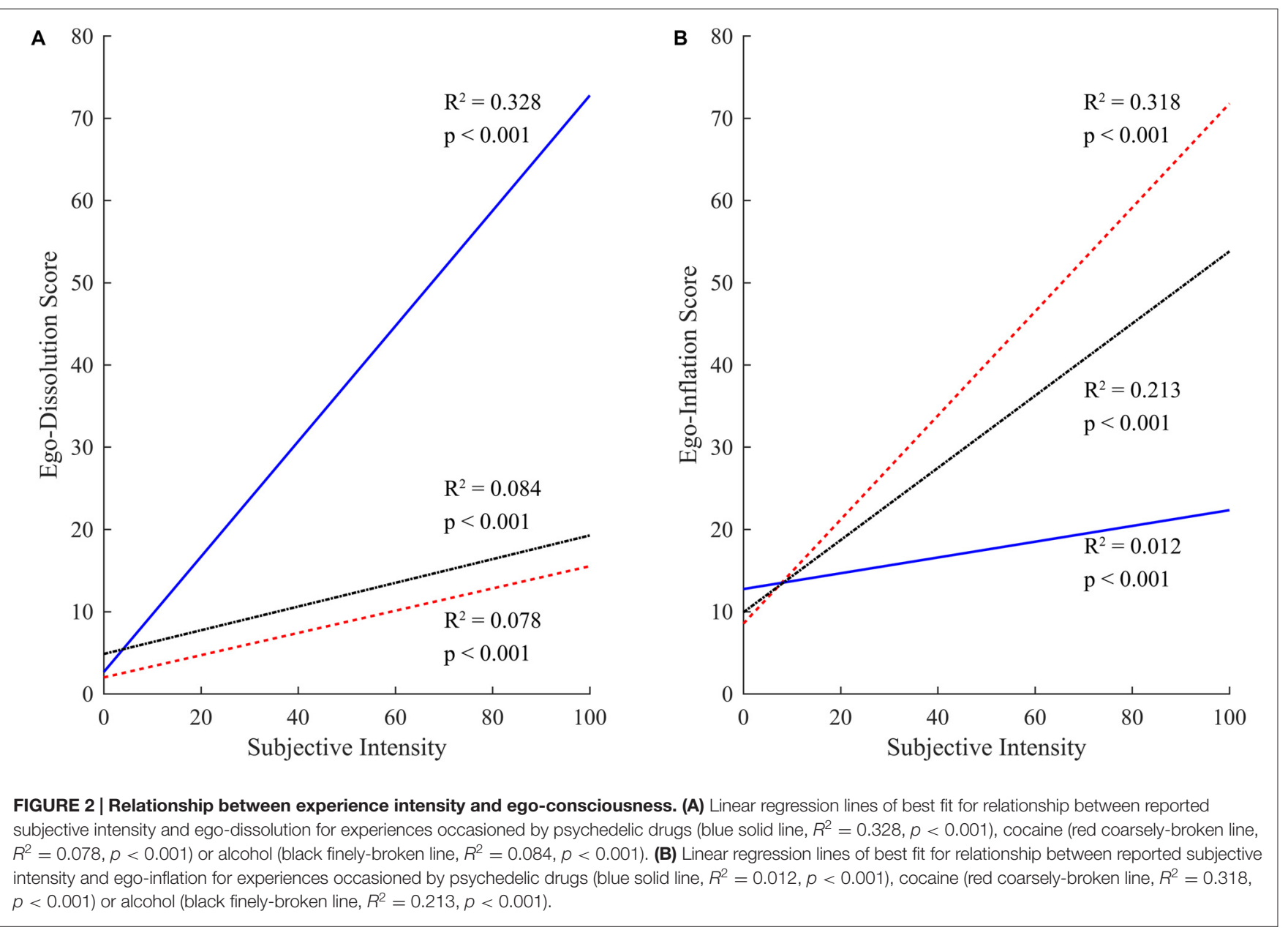

greater than the median response for cocaine $(0=$ "No change", interquartile range $=0$, skewness -0.135 , median time elapsed since experience $=1-5$ years $)$ or alcohol $(0=$ "No change", interquartile range $=0$, skewness $=-0.220$, median time elapsed since experience $=1-4$ weeks; $p<0.001$ for both comparisons, Wilcoxon rank sum test). The difference between the mean change in well-being between cocaine and alcohol was not significant $(p=0.260$, Wilcoxon rank sum test).

There was a trend for a positive correlation between egodissolution score and improvement in well-being across all drug classes, with this effect reaching statistical significance after correction for multiple comparisons only for experiences occasioned by psychedelic drugs (psychedelic: rho $=0.392$ [0.342, 0.442], $p<0.001$, cocaine: rho $=0.103[-0.006,0.204]$, $p=0.045$; alcohol: rho $=0.084[-0.009,0.181], p=0.091)$. This correlation was significantly stronger for psychedelic drugs compared with either cocaine $(Z=5.15, p<0.001)$ or alcohol $(Z=5.63, p<0.001)$, but did not differ between cocaine and alcohol drug experiences $(Z=0.267, p=0.789)$.

Ego-inflation was positively correlated with improvement in well-being for psychedelic drugs (rho $=0.198$ [0.135, 0.263], $p<0.001$ ). There was a trend for a negative correlation between ego-inflation and well-being for experiences with cocaine and alcohol however, although these correlations were nonsignificant after correction for multiple comparisons (Cocaine: rho $=-0.083[-0.198,0.027], p=0.107$. Alcohol: rho $=-0.112$ $[-0.216,-0.014], p=0.024)$. Whilst the correlation between ego-inflation and well-being was significantly different for psychedelic drugs compared with both cocaine $(Z=4.71$, $p<0.001)$ and alcohol $(Z=5.53, p<0.001)$, it was not significantly different between cocaine and alcohol $(Z=0.408$, $p=0.683$ ).

For psychedelic experiences, the positive correlation between ego-dissolution and increase in well-being was stronger than between ego-inflation and change in well-being $\left(t_{(1040)}=5.48\right.$, $p<0.001)$. For both cocaine and alcohol experiences, the negative correlation between ego-inflation and change in wellbeing was stronger than the correlation between ego-dissolution and change in well-being (Cocaine: $t_{(374)}=3.14, p=0.001$. Alcohol: $\left.t_{(405)}=4.13, p<0.001\right)$.

\section{DISCUSSION}

The results presented in this study demonstrate the internal consistency, single-factor psychometric structure and construct validity the EDI, a new 8-item self-report questionnaire designed to measure the experience of ego-dissolution. Additionally, 


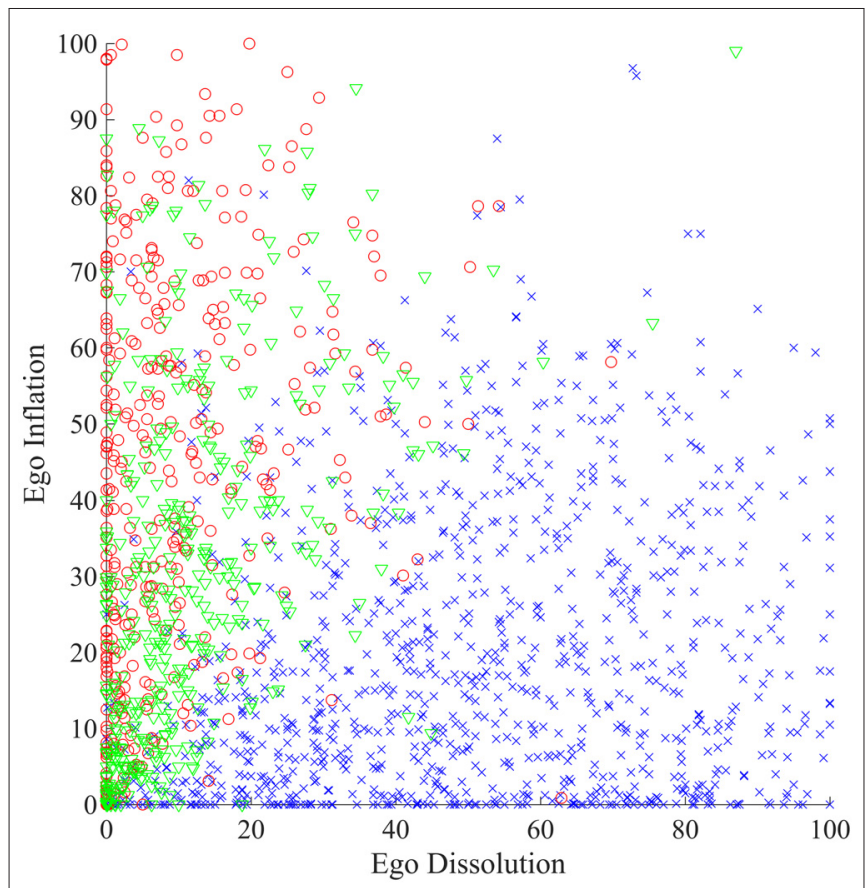

FIGURE 3 | The relationship between ego-dissolution and ego-inflation for experiences occasioned by classical psychedelics (blue crosses), cocaine (red circles) and alcohol (green triangles).

our results demonstrate that ego-dissolution positively correlates with drug dose and experience intensity specifically for psychedelic drugs, compared with cocaine or alcohol experiences. This result mirrors the previously demonstrated positive relationship between psilocybin dose and altered states of consciousness, including "oceanic boundlessness" and "dread of ego dissolution" (as measured by the 5D-ASC questionnaire; Hasler et al., 2004; Wittmann et al., 2007; Studerus et al., 2011) and the mystical experience (Griffiths et al., 2011). Indeed, we also found a positive correlation between psychedelic dose and unitive experience (rho $=0.307, p<0.001$ ).

The experience of a coherent and well-circumscribed self is a cardinal feature of adult human waking consciousness (Carhart-Harris and Friston, 2010; Carhart-Harris et al., 2014). Conversely, the experience of ego-dissolution is unfamiliar to most people, and is related to relatively rare altered states of consciousness, such as the psychedelic experience (Huxley, 1954; Leary et al., 1964; Grof, 1980; Harrison, 2010; CarhartHarris et al., 2014; Lebedev et al., 2015) and the mystical experience (Stace, 1960; James, 1985). Despite the relative rarity of the ego-dissolution experience, a fuller understanding of its neurobiological correlates may inform our understanding of the therapeutic mechanism of action of psychedelic drugs (Grof, 1980; Griffiths et al., 2008, 2011) and human consciousness more generally (Carhart-Harris et al., 2014). The development and validation of the EDI is an important contribution to this research programme.

An understanding of the neurobiological correlates of selfexperience is also of great importance to a number of mental health conditions, where the sense of self is disrupted or compromised (Northoff, 2014). A disrupted sense of self has long been considered to be a core phenomenological feature of acute psychosis (Federn, 1952; Laing, 1959; Fischman, 1983; Jaspers, 1997; Scharfetter, 1981; Nour and Barrera, 2015). Recently this idea has been extended by Sass and Parnas (2003), who propose that the core phenomenological alteration in schizophrenia is an "instability of pre-reflective self-awareness", which has been termed an "ipseity-disorder" or "self-disorder" (Sass et al., 2011). They argue that saturating all subjective experience is a prereflective awareness of self as the unified subject of experience (Sass and Parnas, 2003). This notion is closely related to the feeling of inhabiting a living body embedded in the world (Stanghellini, 2009). As the pre-reflective sense of self is related to a feeling of immersion in a social world, ipseity disturbance may also result in the deficits in social cognition seen in patients (Parnas and Bovet, 1991; Stanghellini, 2001, 2009; Nelson et al., 2009; Nordgaard and Parnas, 2014; Nour and Barrera, 2015). Further research is required to clarify the relationship between the self-disturbances seen in schizophrenia and psychedelic states, as well as the relationship between other features of the psychedelic state and psychosis (Corlett et al., 2009).

Our results represent a necessary step in the demonstration of the construct validity of ego-dissolution. Construct validity can be decomposed into discriminant and convergent validity. Discriminant validity of the EDI was demonstrated by the fact that items relating to ego-dissolution and those relating to ego-inflation loaded onto two orthogonal factors in the exploratory factor analysis. Convergent validity of the EDI was demonstrated by the strong positive correlation between EDI and our (MEQ-based) measure of the unitive experience. This suggests that experiences of ego-dissolution, unity and dissolved ego-boundaries may be conceptually inseparable (Federn, 1952; Savage, 1955; Fischman, 1983), occurring together during "peak" psychedelic experiences. Consistent with this hypothesis, the item "I felt at one with the universe" loaded particularly strongly on the "ego-dissolution" factor (0.830), together with items explicitly referring to "dissolution" and "disintegration" of self or ego (0.883 and 0.897 , respectively).

Our measure of ego-inflation, in contrast to ego-dissolution, showed a significant dose-response relationship with cocaine, but not psychedelics or alcohol. Although all three drug classes showed a positive correlation between ego-inflation and experience intensity, this relationship was strongest for cocaine and weakest for psychedelic drugs. These results suggest that experiences occasioned by cocaine are in some sense antithetical to the psychedelic experience; with cocaine seeming to promote self-centeredness rather than the selflessness associated with psychedelics.

Consistent with this hypothesis, a binary SVM classifier was able to identify experiences occasioned by psychedelic drugs vs. cocaine or alcohol with over $85 \%$ accuracy using the ego-dissolution and ego-inflation scores alone. This machine-learning approach may be usefully applied in a number of contexts. For example, it may help to inform debates over whether hybrid compounds such as 3,4methylenedioxymethamphetamine (MDMA) should be classed as "ego-dissolving" psychedelic-like agents, "ego- 
inflating" stimulant-like agents, both, or neither. Moreover, used in conjunction with neurobiological measures, such as neuroimaging, these tools may help us to identify key (defining) properties of different psychoactive drugs, as well as nondrug-induced states, in a data-driven manner, and may inform novel hypotheses concerning the endogenous role of $5-\mathrm{HT}_{2 \mathrm{~A}}$ receptors. This may help us to address questions regarding the similarities and differences of different altered states of consciousness such as dreaming, psychosis and the psychedelic state (Carhart-Harris, 2007). More generally, our results suggest that the specific way in which a drug disturbs ego-consciousness may inform a novel phenomenologically-based classification system for psychoactive substances.

Subjects in the present study reported that on average their reported experiences with psychedelic drugs had a positive and lasting impact on their well-being, which correlated positively with the degree of ego-dissolution experienced (rho $=0.392$ ). This is consistent with previous work, which has established that mystical (or "peak") experiences occasioned by psilocybin correlate positively with increases in "openness" (MacLean et al., 2011), well-being (Barrett et al., 2015), and the meaningfulness/spiritual significance of the experience (Griffiths et al., 2008). Similarly, one influential model of LSD therapy states that the experience of "ego death and . . loss of boundaries between the subject and the objective world, with ensuing feelings of unity" is of great therapeutic benefit (Grof, 1980). Moreover, lifetime psychedelic use has been associated with reduced odds ratio of psychological distress, suicidality and certain mental health problems in large population samples (Krebs and Johansen, 2013; Hendricks et al., 2015; Johansen and Krebs, 2015).

Regarding the neurobiology of the psychedelic state, previous work has indicated that psychedelics disrupt the integrity of the default-mode network (DMN), a normally well-integrated network of (mostly cortical) brain regions that display high metabolic demands, "connector-hub" status and appear to be involved in high-level functions such as the processing of selfspecific information (Qin and Northoff, 2011; Buckner, 2013; Speth et al., 2016). Psilocybin and DMT-containing ayahuasca decrease functional connectivity between key DMN hub regions (Carhart-Harris et al., 2012; Muthukumaraswamy et al., 2013; Palhano-Fontes et al., 2015). Psilocybin and LSD also disrupt the functional segregation between usually well-demarcated brain networks, promoting increased global integration (CarhartHarris et al., 2013; Roseman et al., 2014), which correlates with ego-dissolution (Tagliazucchi et al., 2016). Disrupted integrity of the DMN and reduced anticorrelation between DMN and task-positive network activity may facilitate a less constrained style of cognition and a weakening of the feeling of a well-circumscribed self. This may be a psychological consequence of a less constrained (more entropic) style of brain activity and a "collapse" in the normal hierarchical organization of cortical circuits, which normally functions to finesse perception and cognition by minimizing uncertainty (Hohwy, 2007; Carhart-Harris and Friston, 2010; Friston, 2010; Carhart-Harris et al., 2014; Roseman et al., 2014; Tagliazucchi et al., 2014; Nour and Nour, 2015).
These previous experimental findings suggest that the integrity of the DMN may be important for normal selfexperience (Qin and Northoff, 2011; Carhart-Harris et al., 2014). Relatively few studies have explicitly investigated the neural correlates of ego-dissolution experiences occasioned by psychedelic drugs. Muthukumaraswamy et al. (2013) found that the experience of ego-disintegration occasioned by psilocybin correlated with decreased alpha power in the posterior cingulate cortex, a DMN hub region, using magnetoencephalography (MEG). Lebedev et al. (2015) by contrast, found that egodissolution correlated with decreased functional connectivity between the anterior parahippocampal cortex and higher-level cortical DMN regions as well as decreased (within-network) integrity of the salience network, and reduced inter-hemispheric communication.

A recent study found that the degree of ego-dissolution occasioned by LSD was correlated with global functional connectivity ("Functional Connectivity Density", as measured by functional magnetic resonance imaging (fMRI)) in bilateral temporo-parietal junction (angular gyrus) and bilateral insular cortex (Tagliazucchi et al., 2016). More generally, this study reported that LSD induced increases in global connectivity in more widespread high-level association cortices, which overlap substantially with the default mode network. A separate analysis of the same fMRI data further revealed that egodissolution showed a strong inverse correlation with DMN network integrity (measured as within-network resting state functional connectivity), and functional connectivity between the parahippocampus and retrosplenial cortex (CarhartHarris et al., 2016). Moreover, significant relationships were found between ego-dissolution and decreased delta and alpha power (e.g., in posterior cingulate cortex) as measured by MEG (Carhart-Harris et al., 2016), replicating previous findings with psilocybin (Muthukumaraswamy et al., 2013).

One limitation of these studies is that they either used a single-item measure of ego-dissolution (Muthukumaraswamy et al., 2013; Carhart-Harris et al., 2016; Tagliazucchi et al., 2016), or principle component analysis loadings from an (as yet) unvalidated questionnaire which included items indirectly related to ego-dissolution (e.g., perceptual abnormalities; Lebedev et al., 2015). Thus, the validated EDI allows for a more rigorous study of the neural correlates of ego-dissolution experienced in a number of altered states of consciousness, including those associated with psychedelic drugs and spiritual practice.

The present study has some limitations. Firstly, the population sampled was fairly homogenous, which limits the study's external validity, and thus our ability to extrapolate to a broader demographic. Specifically, most of our subjects were male, under the age of 30 , and had at least some university education. Over half the subjects had used classical psychedelic drugs on over 10 occasions. This also raises the possibility that our subjects' responses were biased by their familiarity with reports about paradigmatic features of the psychedelic experience, such as ego-dissolution. Moreover, we did not collect information on the setting in which the psychedelic 
experiences took place, which is known to influence the quality of the experience (Leary et al., 1964; Grof, 1976; Fischman, 1983). Another important limitation of our study is its retrospective design, which introduces potential inaccuracies in experience recall. We employed an anonymous internet questionnaire design to facilitate the collection of a large data sample of subjects from around the world. Although this approach has its strengths, it is impossible to verify that the experiences attributed to psychedelics were indeed caused by these substances. Finally, we asked subjects to estimate the dose of psychedelic ingested using "LSD-equivalent" doses, so as to more easily investigate dose-response relationships across different classical psychedelics. The inaccuracy introduced by this approach, however, is likely to have weakened, rather than strengthened, any observed dose-response relationship between psychedelics and ego-dissolution.

Given these limitations, future studies should include a more heterogeneous sampling population, perhaps by intentionally recruiting subjects from different cultural and religious backgrounds, to explore the influence of these factors on ego-dissolution. It would also be of interest to investigate the relationship between responses on the EDI and other validated scales, for example the 5D-ASC (Studerus et al., 2010). Finally, experimental studies in which a range of psychedelic drug doses are administered to subjects in a blinded manner would be able to assess how subject-specific factors influence the relationship between dose and subjective effects, whilst controlling for recall effects and the effect of setting.

\section{REFERENCES}

Barrett, F. S., Johnson, M. W., and Griffiths, R. R. (2015). Validation of the revised mystical experience questionnaire in experimental sessions with psilocybin. J. Psychopharmacol. 29, 1182-1190. doi: 10.1177/02698811156 09019

Bleuler, E. (1950). Dementia Praecox or The Group of Schizophrenias. New York, NY: International University Press. (Originally published in 1911).

Buckner, R. L. (2013). The brain's default network: origins and implications for the study of psychosis. Dialogues Clin. Neurosci. 15, 351-358.

Budaev, S. V. (2010). Using principal components and factor analysis in animal behaviour research: caveats and guidelines. Ethology 116, 472-480. doi: 10. 1111/j.1439-0310.2010.01758.x

Carhart-Harris, R. (2007). Waves of the unconscious: the neurophysiology of dreamlike phenomena and its implications for the psychodynamic model of the mind. Neuropsychoanal. 9, 183-211. doi: 10.1080/15294145.2007.107 73557

Carhart-Harris, R. L., Erritzoe, D., Williams, T., Stone, J. M., Reed, L. J., Colasanti, A., et al. (2012). Neural correlates of the psychedelic state as determined by fMRI studies with psilocybin. Proc. Natl. Acad. Sci. U S A 109, 2138-2143. doi: 10.1073/pnas.1119598109

Carhart-Harris, R. L., and Friston, K. J. (2010). The default-mode, ego-functions and free-energy: a neurobiological account of Freudian ideas. Brain 133, 1265-1283. doi: 10.1093/brain/awq010

Carhart-Harris, R. L., Leech, R., Erritzoe, D., Williams, T. M., Stone, J. M., Evans, J., et al. (2013). Functional connectivity measures after psilocybin inform a novel hypothesis of early psychosis. Schizophr. Bull. 39, 1343-1351. doi: 10. 1093/schbul/sbs117

Carhart-Harris, R. L., Leech, R., Hellyer, P. J., Shanahan, M., Feilding, A., Tagliazucchi, E., et al. (2014). The entropic brain: a theory of conscious states informed by neuroimaging research with psychedelic drugs. Front. Hum. Neurosci. 8:20. doi: 10.3389/fnhum.2014.00020
In conclusion, the present study offers initial-phase validation of the EDI, and adds to the growing evidence that ego-dissolution is a key phenomenological feature of the psychedelic experience, which may be studied experimentally. The existence of the EDI will facilitate future research into the neural correlates of this experience, which is of relevance for psychedelic-assisted psychotherapy and the phenomenology of certain psychiatric conditions.

\section{AUTHOR CONTRIBUTIONS}

MMN and RLC-H conceived of and designed this study and interpreted the results; contributed to drafting the work and revised it critically for important intellectual content. All authors approved the final version of this manuscript to be published and agreed to be accountable for all aspects of the work in ensuring that questions related to the accuracy or integrity of any part of the work be appropriately investigated and resolved. MMN and LE undertook statistical analysis of the data. LE managed the implementation of the questionnaire and subsequent data collection. MMN wrote the article, with editing from RLC-H.

\section{FUNDING}

MMN is funded by the Medical Research Council, UK. RLC-H is funded by Mosley Foundation. DN is funded by Safra Foundation.

Carhart-Harris, R. L., Murphy, K., Leech, R., Erritzoe, D., Wall, M. B. Ferguson, B., et al. (2015). The effects of acutely administered 3,4-methylenedioxymethamphetamine on spontaneous brain function in healthy volunteers measured with arterial spin labeling and blood oxygen level-dependent resting state functional connectivity. Biol. Psychiatry 78, 554-562. doi: 10.1016/j.biopsych.2013.12.015

Carhart-Harris, R. L., Muthukumaraswamy, S., Roseman, L., Kaelen, M., Droog, W., and Nutt, D. J. (2016). Neural correlates of the LSD experience revealed by multimodal neuroimaging. Proc. Natl. Acad. Sci. U S A 113, 4853-4858. doi: 10.1073/pnas.1518377113

Cattell, R. (1966). Scree test for number of factors. Multivariate Behav. Res. 1, 245-276. doi: 10.1207/s15327906mbr0102_10

Corlett, P. R., Frith, C. D., and Fletcher, P. C. (2009). From drugs to deprivation: a Bayesian framework for understanding models of psychosis. Psychopharmacology (Berl) 206, 515-530. doi: 10.1007/s00213-009-1561-0

Cronbach, L. (1951). Coefficient $\alpha$ and the internal structure of tests. Psychometrika 16, 297-333. doi: 10.1007/bf02310555

Dittrich, A. (1998). The standardized psychometric assessment of altered states of consciousness (ASCs) in humans. Pharmacopsychiatry 31, 80-84. doi: 10 . 1055/s-2007-979351

Eveloff, H. H. (1968). The LSD syndrome. A review. Calif. Med. 109, 368-373.

Federn, P. (1952). Ego Psychology and the Psychoses. New York, NY: Basic Books.

Field, A. (2013). Discovering Statistics Using IBM SPSS Statistics, 4th Edn. London: SAGE Publications Ltd.

Fischman, L. G. (1983). Dreams, hallucinogenic drug states and schizophrenia: a psychological and biological comparison. Schizophr. Bull. 9, 73-94. doi: 10. 1093/schbul/9.1.73

Friston, K. (2010). The free-energy principle: a unified brain theory? Nat. Rev. Neurosci. 11, 127-138. doi: 10.1038/nrn2787

Goodman, N. (2002). The serotonergic system and mysticism: could LSD and the nondrug-induced mystical experience share common neural mechanisms? J. Psychoactive Drugs 34, 263-272. doi: 10.1080/02791072.2002.10399962 
Griffiths, R. R., Johnson, M. W., Richards, W. A., Richards, B. D., McCann, U., and Jesse, R. (2011). Psilocybin occasioned mystical-type experiences: immediate and persisting dose-related effects. Psychopharmacology (Berl) 218, 649-665. doi: 10.1007/s00213-011-2358-5

Griffiths, R. R., Richards, W. A., Johnson, M. W., McCann, U. D., and Jesse, R. (2008). Mystical-type experiences occasioned by psilocybin mediate the attribution of personal meaning and spiritual significance 14 months later. J. Psychopharmacol. 22, 621-632. doi: 10.1177/0269881108094300

Grof, S. (1976). Realms of the Human Unconscious. Observations from LSD Research. New York, NY: E.P. Dutton.

Grof, S. (1980). LSD Psychotherapy. Alameda, CA: Hunter House Publishers.

Harrison, J. (2010). Ego death and psychedelics. MAPS Bull. 20, 40-41.

Hasler, F., Grimberg, U., Benz, M. A., Huber, T., and Vollenweider, F. X. (2004). Acute psychological and physiological effects of psilocybin in healthy humans: a double-blind, placebo-controlled dose? effect study. Psychopharmacology (Berl.) 172, 145-156. doi: 10.1007/s00213-003-1640-6

He, H., and Garcia, E. A. (2009). Learning from imbalanced data. IEEE Trans. Knowl. Data Eng. 21, 1263-1284. doi: 10.1109/tkde.2008.239

Hendricks, P. S., Thorne, C. B., Clark, C. B., Coombs, D. W., and Johnson, M. W. (2015). Classic psychedelic use is associated with reduced psychological distress and suicidality in the United States adult population. J. Psychopharmacol. 29, 280-288. doi: 10.1177/0269881114565653

Hohwy, J. (2007). The sense of self in the phenomenology of agency and perception. Psyche 13, 1-20.

Hood, R. (1975). The construction and preliminary validation of a measure of reported mystical experience. J. Sci. Study Relig. 14, 29-41. doi: 10. $2307 / 1384454$

Horn, J. (1965). A rationale and test for the number of factors in factor analysis. Psychometrika 30, 179-185. doi: 10.1007/bf02289447

Huxley, A. (1954). The Doors of Perception: and Heaven and Hell. London: Vintage.

James, W. (1882). Subjective effects of nitrous oxide. Mind 7.

James, W. (1985). The Varieties of Religious Experience: A Study in Human Nature. London: Penguin Classics. (Originally published in 1902).

Jaspers, K. (1997). General Psychopathology. Baltimore, MA: The Johns Hopkins University Press. (Originally published in 1913).

Johansen, P.- Ø., and Krebs, T. S. (2015). Psychedelics not linked to mental health problems or suicidal behavior: a population study. J. Psychopharmacol. 29, 270-279. doi: 10.1177/0269881114568039

Johnson, M. W., Garcia-Romeu, A., Cosimano, M. P., and Griffiths, R. R. (2014). Pilot study of the 5-HT2AR agonist psilocybin in the treatment of tobacco addiction. J. Psychopharmacol. 28, 983-992. doi: 10.1177/0269881114548296

Johnson, M. M., Richards, W., and Griffiths, R. R. (2008). Human hallucinogen research: guidelines for safety. J. Psychopharmacol. 22, 603-620. doi: 10 . $1177 / 0269881108093587$

Klee, G. D. (1963). Lysergic acid diethylamide (LSD-25) and ego functions. Arch. Gen. Psychiatry 8, 461-474. doi: 10.1001/archpsyc.1963.01720110037005

Krebs, T. S., and Johansen, P.-Ø. (2013). Psychedelics and mental health: a population study. PLoS One 8:e63972. doi: 10.1371/journal.pone.0063972

Laing, R. (1959). The Divided Self. An Existential Study in Sanity and Madness. London: Penguin Books.

Leary, T., Metzner, R., and Alpert, R. (1964). The Psychedelic Experience: Manual Based on the Tibetan Book of the Dead. London: Penguin Classics.

Lebedev, A. V., Lövdén, M., Rosenthal, G., Feilding, A., Nutt, D. J., and CarhartHarris, R. L. (2015). Finding the self by losing the self: neural correlates of ego-dissolution under psilocybin. Hum. Brain Mapp. 36, 3137-3153. doi: 10. 1002/hbm.22833

Lyvers, M., and Meester, M. (2012). Illicit use of LSD or psilocybin, but not MDMA or nonpsychedelic drugs, is associated with mystical experiences in a dosedependent manner. J. Psychoactive Drugs 44, 410-417. doi: 10.1080/02791072. 2012.736842

MacLean, K. A., Johnson, M. W., and Griffiths, R. R. (2011). Mystical experiences occasioned by the hallucinogen psilocybin lead to increases in the personality domain of openness. J. Psychopharmacol. 25, 1453-1461. doi: 10. $1177 / 0269881111420188$

MacLean, K. A., Leoutsakos, J. M., Johnson, M. W., and Griffiths, R. R. (2012). Factor analysis of the mystical experience questionnaire: a study of experiences occasioned by the hallucinogen. J. Sci. Study Relig. 51, 721-737. doi: 10.1111/j. 1468-5906.2012.01685.x
Muthukumaraswamy, S. D., Carhart-Harris, R. L., Moran, R. J., Brookes, M. J., Williams, T. M., Errtizoe, D., et al. (2013). Broadband cortical desynchronization underlies the human psychedelic state. J. Neurosci. 33, 15171-15183. doi: 10.1523/jneurosci.2063-13.2013

Nelson, B., Sass, L. A., Thompson, A., Yung, A. R., Francey, S. M., Amminger, G. P., et al. (2009). Does disturbance of self underlie social cognition deficits in schizophrenia and other psychotic disorders? Early Interv. Psychiatry 3, 83-93. doi: 10.1111/j.1751-7893.2009.00112.x

Nordgaard, J., and Parnas, J. (2014). Self-disorders and the schizophrenia spectrum: a study of 100 first hospital admissions. Schizophr. Bull. 40, 1300-1307. doi: 10.1093/schbul/sbt239

Northoff, G. (2014). How is our self altered in psychiatric disorders? A neurophenomenal approach to psychopathological symptoms. Psychopathology 47, 365-376. doi: 10.1159/000363351

Nour, M. M., and Barrera, A. (2015). Schizophrenia, subjectivity and mindreading. Schizophr. Bull. 41, 1214-1219. doi: 10.1093/schbul/sbv035

Nour, M. M., and Nour, J. M. (2015). Perception, illusions and Bayesian inference. Psychopathology 48, 217-221. doi: 10.1159/000437271

O'Connor, B. P. (2000). SPSS and SAS programs for determining the number of components using parallel analysis and velicer's MAP test. Behav. Res. Methods Instrum. Comput. 32, 396-402. doi: 10.3758/bf03200807

Palhano-Fontes, F., Andrade, K., Tofoli, L., Santos, A. C., Crippa, J. A., Hallak, J. E., et al. (2015). The psychedelic state induced by ayahuasca modulates the activity and connectivity of the default mode network. PLoS One 10:e0118143. doi: 10. 1371/journal.pone.0118143

Parnas, J. (2011). A disappearing heritage: the clinical core of schizophrenia. Schizophr. Bull. 37, 1121-1130. doi: 10.1093/schbul/sbr081

Parnas, J., and Bovet, P. (1991). Autism in schizophrenia revisited. Compr. Psychiatry 32, 7-21. doi: 10.1016/0010-440x(91)90065-k

Parnas, J., Møller, P., Kircher, T., Thalbitzer, L., Jansson, L., Handest, P., et al. (2005). EASE: examination of anomalous self-experience. Psychopathology 38, 236-258. doi: 10.1159/000088441

Qin, P., and Northoff, G. (2011). How is our self related to midline regions and the default-mode network? Neuroimage 57, 1221-1233. doi: 10.1016/j.neuroimage. 2011.05.028

Roseman, L., Leech, R., Feilding, A., Nutt, D. J., and Carhart-Harris, R. L. (2014). The effects of psilocybin and MDMA on between-network resting state functional connectivity in healthy volunteers. Front. Hum. Neurosci. 8:204. doi: 10.3389/fnhum.2014.00204

Sass, L. A., and Parnas, J. (2003). Schizophrenia, consciousness and the self. Schizophr. Bull. 29, 427-444. doi: 10.1093/oxfordjournals.schbul.a007017

Sass, L., Parnas, J., and Zahavi, D. (2011). Phenomenological psychopathology and schizophrenia: contemporary approaches and misunderstandings. Philos. Psychiatr. Psychol. 18, 1-23. doi: 10.1353/ppp.2011.0008

Savage, C. (1955). Variations in ego feeling induced by D-lysergic acid diethylamide (LSD-25). Psychoanal. Rev. 42, 1-16.

Scharfetter, C. (1981). Ego-psychopathology: the concept and its empirical evaluation. Psychol. Med. 11, 273-280. doi: 10.1017/s0033291700052090

Schmid, Y., Enzler, F., Gasser, P., Grouzmann, E., Preller, K. H., Vollenweider, F. X., et al. (2015). Acute effects of lysergic acid diethylamide in healthy subjects. Biol. Psychiatry 78, 544-553. doi: 10.1016/j.biopsych.2014. 11.015

Speth, J., Speth, C., Kaelen, M., Schloerscheidt, A. M., Feilding, A., Nutt, D. J., et al. (2016). Decreased mental time travel to the past correlates with default-mode network disintegration under lysergic acid diethylamide. J. Psychopharmacol. 30, 344-353. doi: 10.1177/0269881116628430

Stace, W. (1960). Mysticism and Philosophy. New York, NY: MacMillan Press.

Stanghellini, G. (2001). Psychopathology of common sense. Philos. Psychiatr. Psychol. 8, 201-218. doi: 10.1353/ppp.2001.0018

Stanghellini, G. (2009). Embodiment and schizophrenia. World Psychiatry 8, 56-59. doi: 10.1002/j.2051-5545.2009.tb00212.x

Strassman, R. J., Qualls, C. R., Uhlenhuth, E. H., and Kellner, R. (1994). Doseresponse study of N,N-dimethyltryptamine in humans. II. Subjective effects and preliminary results of a new rating scale. Arch. Gen. Psychiatry 51, 98-108. doi: 10.1001/archpsyc.1994.03950020022002

Studerus, E., Gamma, A., Kometer, M., and Vollenweider, F. X. (2012). Prediction of psilocybin response in healthy volunteers. PLoS One 7:e30800. doi: 10 1371/journal.pone.0030800 
Studerus, E., Gamma, A., and Vollenweider, F. X. (2010). Psychometric evaluation of the altered states of consciousness rating scale (OAV). PLoS One 5:e12412. doi: 10.1371/journal.pone.0012412

Studerus, E., Kometer, M., Hasler, F., and Vollenweider, F. X. (2011). Acute, subacute and long-term subjective effects of psilocybin in healthy humans: a pooled analysis of experimental studies. J. Psychopharmacol. 25, 1434-1452. doi: 10.1177/02698811103 82466

Tagliazucchi, E., Carhart-Harris, R., Leech, R., Nutt, D., and Chialvo, D. R. (2014). Enhanced repertoire of brain dynamical states during the psychedelic experience. Hum. Brain Mapp. 35, 5442-5456. doi: 10.1002/hbm. 22562

Tagliazucchi, E., Roseman, L., Kaelen, M., Orban, C., Muthukumaraswamy, S. D., Murphy, K., et al. (2016). Increased global functional connectivity correlates with LSD-induced ego dissolution. Curr. Biol. 26, 1043-1050. doi: 10.1016/j. cub.2016.02.010

Trichter, S., Klimo, J., and Krippner, S. (2009). Changes in spirituality among ayahuasca ceremony novice participants. J. Psychoactive Drugs 41, 121-134. doi: $10.1080 / 02791072.2009 .10399905$
Vollenweider, F. X., and Kometer, M. (2010). The neurobiology of psychedelic drugs: implications for the treatment of mood disorders. Nat. Rev. Neurosci. 11, 642-651. doi: 10.1038/nrn2884

Wittmann, M., Carter, O., Hasler, F., Cahn, B. R., Grimberg, U., Spring, P., et al. (2007). Effects of psilocybin on time perception and temporal control of behaviour in humans. J. Psychopharmacol. 21, 50-64. doi: 10. $1177 / 0269881106065859$

Conflict of Interest Statement: The authors declare that the research was conducted in the absence of any commercial or financial relationships that could be construed as a potential conflict of interest.

Copyright (c) 2016 Nour, Evans, Nutt and Carhart-Harris. This is an open-access article distributed under the terms of the Creative Commons Attribution License (CC BY). The use, distribution and reproduction in other forums is permitted, provided the original author(s) or licensor are credited and that the original publication in this journal is cited, in accordance with accepted academic practice. No use, distribution or reproduction is permitted which does not comply with these terms. 